\title{
Recent result of gyrotron operation in NIFS
}

\author{
Satoshi Ito ${ }^{1, a}$, Takashi Shimozuma ${ }^{1}$, Yasuo Yoshimura ${ }^{1}$, Hiroe Igami ${ }^{1}$, Hiromi Takahashi ${ }^{1}$, Masaki Nishiura ${ }^{2}$, Sakuji \\ Kobayashi ${ }^{1}$, Yoshinori Mizuno ${ }^{1}$, Kota Okada ${ }^{1}$ and Shin Kubo ${ }^{1}$ \\ ${ }^{1}$ National Institute of Fusion Science, ECH Group, 322-6 Oroshi-cho Toki, Gifu, Japan \\ ${ }^{2}$ University of Tokyo, Graduate school, 5-1-5 Kashiwa-no-ha Kashiha-shi, Chiba, Japan
}

\begin{abstract}
In the last Large Helical Device (LHD) experimental campaign, a $154 \mathrm{GHz}$ gyrotron which had been conditioned to generate $1 \mathrm{MW} / 2 \mathrm{~s}, 0.5 \mathrm{MW} / \mathrm{CW}$ was installed for LHD experiments. Four high power gyrotrons (three-77 GHz/1 1.5 MW and one-154 GHz/1 MW) and a CW gyrotron (84 GHz/0.2 MW) are ready. Our experiment requires high energy and various injection patterns for Electron Cyclotron Resonance Heating (ECRH). Higher individual injection power and various injection patterns, we developed a power enhancement method by stepped anode acceleration voltage control and operated the gyrotron in the hard excitation region. These operations were realized by a remote controlled waveform generator. However the oscillation map of high power or long pulse operation in the hard excitation region were limited because in order to achieve the hard excitation region by the anode voltage control one must pass through the high anode current phase within a time short enough that the anode or the anode power supply is not overloaded. This limitation becomes more critical when the gyrotron beam current is increased in order to increase the output power. In the long pulse operation it was impossible to reach the hard excitation region in a low beam current $(<10 \mathrm{~A})$.
\end{abstract}

\section{Introduction}

A gyrotron is a kind of a vacuum tube, which is used as an amplifier, a controller of high voltage, an accelerator, or heating devices of plasma. The gyrotron has an important role in plasma experiments, such as ignition or additional heating. Recent plasma physics require ECRH for producing high potential plasma. Therefore gyrotrons have been developed as a power source with several frequencies that are suitable for heating a magnetic confined plasma. Modern gyrotrons can produce millimeter wave with more than $1 \mathrm{MW}$ output power and work as continuous wave $(\mathrm{CW})$. But gyrotrons expects to be the device that needed to produce much more power in the experiments.

The plasma experimental device LHD in National Institute for Fusion Science (NIFS) has the ECRH system, which is one of three heating devices. The ECRH system consists of gyrotrons, power supplies, transmission lines, launchers, and controllers. Gyrotrons generate heating power and the power is transmit through the transmission line including waveguide, miterbends, and polarizer in evacuated condition. Finally, the transmitted microwave that has a proper shape to couple with plasma is launched to the LHD to heat the electrons in localised of plasma. The heating to electrons in the magnetic field is the most important feature of ECRH. The ECRH system is shown in Table 1. We have seven gyrotrons and their transmission lines. Six gyrotrons are always available to operate in both pulsed and CW. The transmission lines are acceptable to transmit high power microwave that diffracts heat load, because the load is removed by cooling jacket with which covered around waveguide. We have improved the high power gyrotrons to perform LHD experiments, but we expect that the new gyrotrons can oscillate more power, thus we have improved a new operation method.

Recent gyrotrons are needed to work in high efficiency because it is difficult to remove the heat load dispersed in the gyrotrons when the gyrotrons are operated at high power. Their oscillation efficiency are requested to be approximately $50 \%$, thus the gyrotron has been developed and aimed at high efficiency operation for a long time and the power supply has also been developed to fulfil the specification of gyrotrons. Figure 2 shows a conventional cavity typed triode gyrotron and power supply in NIFS. The gyrotron is composed of connected to three parts from three kinds of power supplies. Anode voltage (Va) applies between the anode and cathode. Body voltage $(\mathrm{Vb})$ applies between gyrotron body and the cathode. $\mathrm{Vb}$ and magnetic field around the cavity determines the oscillation mode and efficiency. The collector (Cathode) voltage (Vc) is connected between the cathode and the collector and the current flowing between them determines Ic. The collector connects to the ground and applies reverse voltage

\footnotetext{
a Corresponding author: ito@nifs.ac.jp
} 


\begin{tabular}{|c|c|c|c|c|c|}
\hline Power Supply & Frequency $(\mathrm{GHz})$ & Power (MW) & Transmission & Launcher & \\
\hline \multirow{2}{*}{$\begin{array}{l}\text { Solid State } \\
65 \mathrm{kV} / 126 \mathrm{~A}\end{array}$} & 77-\#3-Toshiba & $1.5 / 0.3 \mathrm{cw}$ & $88.9 \mathrm{~mm}$-evacuated & Perp-Upper & \\
\hline & 77-\#2-Toshiba & $1.2 / 0.3 \mathrm{cw}$ & $88.9 \mathrm{~mm}$-evacuated & Perp-Upper & $\rightarrow$ Horizontal \\
\hline \multirow{3}{*}{$\begin{array}{l}\text { Solid State } \\
65 \mathrm{kV} / 126 \mathrm{~A}\end{array}$} & 154-\#1-Toshiba & $1 / 0.5 \mathrm{cw}$ & $88.9 \mathrm{~mm}$-evacuated & Horizontal & \\
\hline & 154-\#2-Toshiba & $1.2 / 0.5 \mathrm{cw}$ & $88.9 \mathrm{~mm}$-evacuated & Horizontal & Under installation \\
\hline & 84-Gycom & $0.5 / 0.1 \mathrm{cw}$ & $31.75 \mathrm{~mm}$-evacuated & Perp-Lower & \\
\hline SS 65kV / 126A & 77-\#1-Toshiba & $1 / 0.3 \mathrm{cw}$ & $88.9 \mathrm{~mm}$-evacuated & Horizontal & \\
\hline Triode $80 \mathrm{kV} / 50 \mathrm{~A}$ & 82.7-Gycom & 0.5 & $88.9 \mathrm{~mm}$ & Perp-Upper & $\rightarrow$ evacuated \\
\hline
\end{tabular}

Table 1 ECRH system

against the $\mathrm{Vb}$ in order to depress the potential $\mathrm{Vc}$. It relieves the heat load by diffracted electrons in the collector. This method is called as Collector Potential Depression (CPD). CPD is also effective in improving the oscillation efficiency of the gyrotron and the efficiency determined by the ratio of input electrical power (the product of $\mathrm{Vc}$ and Ic) and the gyrotron output power. Normally the average efficiency of our gyrotrons is approximately $40 \%$. This power supply is designed in both the pulse operation in the high Ic and the CW operation in low Ic. The current capacities of the body and the anode supplies are $100 \mathrm{~mA}$ respectively. It is also set as the protection to stop operation of the gyrotron. As may be seen in Table 1, two or three gyrotrons are connected to one power supply but it is also possible to operate one gyrotron by use of a high voltage switch set in each power supply. For example, when the gyrotron conditioning is applying, the high voltage on other gyrotrons is turned off through the switch to operate it alone. Output power from the gyrotron is transmitted to the load absorbing all the power with the cooling water.

\section{High power operation}

The output power of the gyrotron is determined by the Ic, $\mathrm{Vb}$, the magnetic field around the cavity, and the pitch factor $\alpha$ which is the velocity ratio of perpendicular and parallel to the magnetic field. The specification of gyrotrons is determined by these factors, and we use them properly for various experiments. For example, triode gyrotron\#3 made by Toshiba is designed for $1.5 \mathrm{MW}$ output power at $77 \mathrm{GHz}$ of frequency, which is suitable to heat the magnetic confined plasma with a $2.75 \mathrm{~T}$ magnetic field. The gyrotron\#3 needs $80 \mathrm{kV}$ of $\mathrm{Vb}, 60 \mathrm{~A}$ of Ic and has $45 \%$ of oscillation efficiency. However sometimes the specification is not achieved, especially in the value of efficiency. The reason is considered in many points but it is difficult to improve because the same thing might occur again after that. Therefore we attempt to raise the oscillation efficiency and output power. Some ways to
All the lines are evacuated in order to avoid the arcing by the high power microwave, and the load is also used in the vacuum condition. The temperature of cooling water in the load is useful to measure the inlet power by the Resistance Temperature Detector (RTD) set between the in and out water flowing paths. The RTD is accurate, thus many loads like this (called as water load) use it. Some company have developed many kinds of water load, but they have both advantages and disadvantages. Therefore, we have developed a suitable water load for our ECRH system.

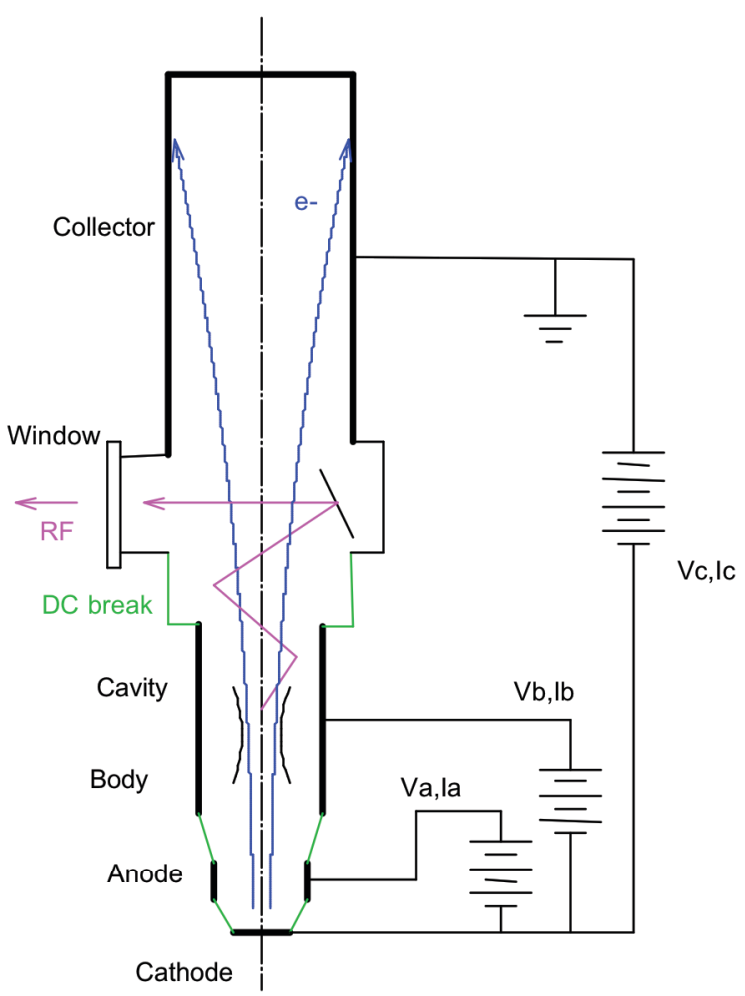

Figure 2. $77 \mathrm{GHz}, 1.5 \mathrm{MW}$ gyrotron with power supply 


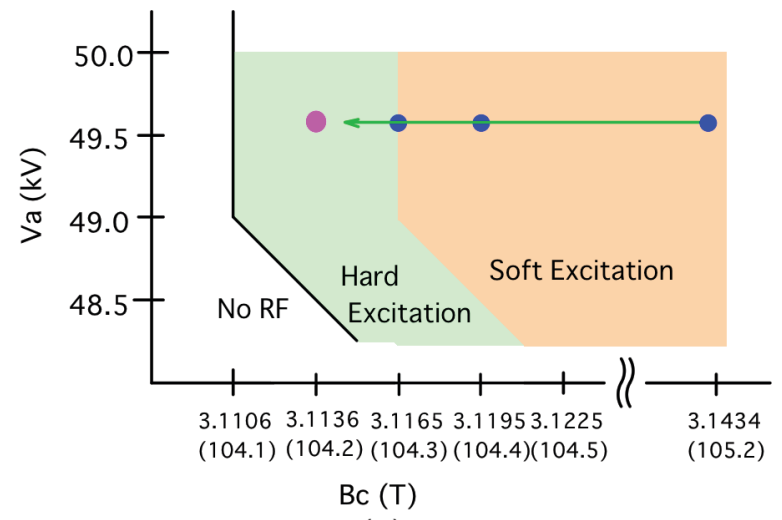

(A)

Figure 3. The oscillation region of $77 \mathrm{GHz} \# 3$ gyrotron at $\mathrm{Ic}=16 \mathrm{~A}$. The region is determined by the cavity magnetic field $(\mathrm{Bc})$ and the anode voltage $(\mathrm{Va})$.

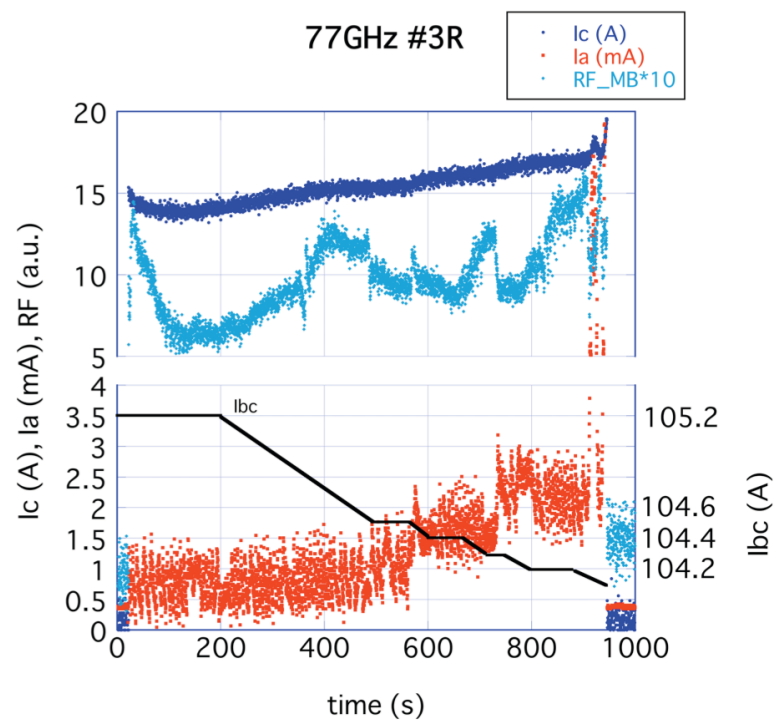

Figure 4. Ic, Ia and RF signal during $\mathrm{CW}$ operation in and out of the hard-excitation region

raise the power are considered, and the starting oscillation in the hard-excitation region is one way to solve this. It is simple to enter the region by changing one operational parameter. Two methods of oscillation in this region exist, the results are explained below.

\subsection{The Result of oscillation by $\mathrm{Bc}$ change in a Hard-excitation region (1)}

One method of oscillation in a hard-excitation region is changing the operational region by the decreasing magnetic field of the cavity [1]. This region is shown in Figure 3 . Normal operation in the stable starting region called the soft-excitation region exists in the blue dots in the orange area in Figure 3. The pink dot is the region that is hardly excited to start the oscillation by the magnetic field. But it is possible to access the region by

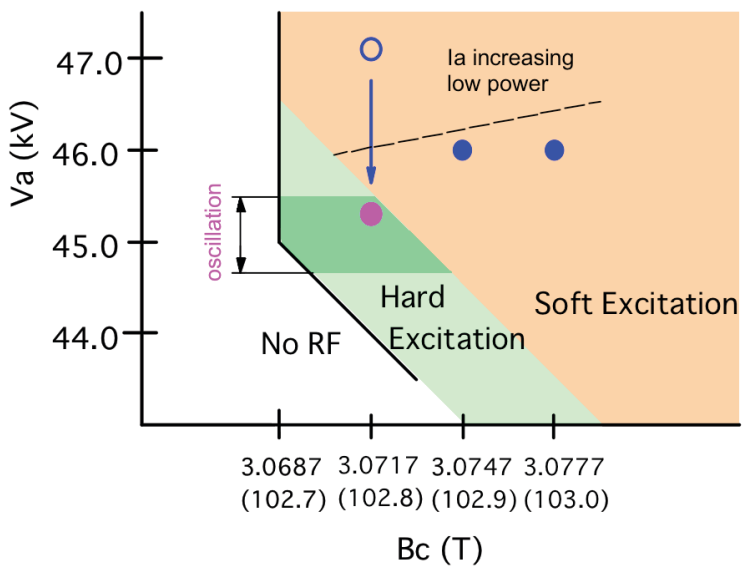

(A)

Figure 5. The oscillation region of the $77 \mathrm{GHz} \# 3$ gyrotron at $\mathrm{Ic}=43 \mathrm{~A}$

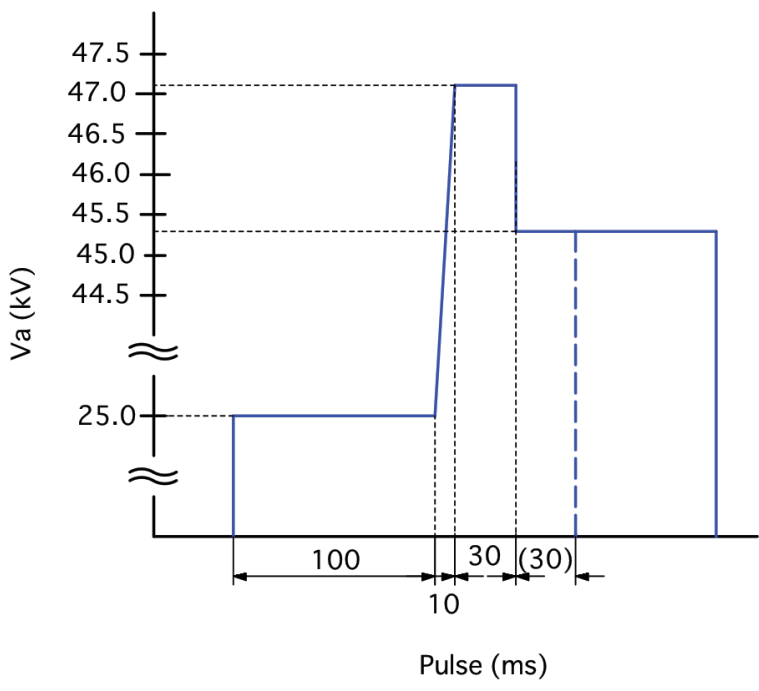

Figure 6. The typical waveform of Va to the enter hardexcitation region at $\mathrm{Ic}=43 \mathrm{~A}$.

the decreasing magnetic field during oscillation. If the operation region of the gyrotron attaches to the edge of No RF, the oscillation is terminated but it is recovered by increasing the field to the border of soft and hardexcitation. That means the oscillation mode is easy to change by magnetic field. This operation is used on the $\mathrm{CW}$ with real-time remote controller of the magnetic field, and output power is measured at the water load.

The result of this operation with $\mathrm{Ic}=15 \mathrm{~A}$ is shown in Figure 4. This oscillation is started from stable softexcitation region at the current of the magnetic field $(\mathrm{Ibc})=105.2 \mathrm{~A}(3.143 \mathrm{~T})$ and the $\mathrm{Va}$ is set to slightly higher value or magnetic field around gun is set a little higher. After the stability of fluctuation Ic at 200s operation, magnetic field is gradually decreased by the $1 \mathrm{~A} /$ min speed rate. We always checked the power at each Ibc, but it could not be operated under Ibc $=104.1 \mathrm{~A}$. Trials at less than $\mathrm{Ibc}=104.1 \mathrm{~A}$ is failed by stopping oscillation. 
As Figure 4 shows that the anode current Ia suddenly rises, which meaning it reached the edge of oscillation. The limit of oscillation in the soft-excitation region was $\mathrm{Ibc}=104.4 \mathrm{~A}$ at pulse operation. Therefore, this means that the operation could enter the hard-excitation region. Acquired power by entering hard-excitation was obtained as $0.381 \mathrm{MW}$ that was improved by $6.7 \%$ compared to the soft-excitation region in the same Ic. And oscillation efficiency was improved to approximately $3 \%$.

We tried this operation at a lower power (lower Ic=7A), but it was impossible to oscillate in the hardexcitation region. The operational region in low power is small in the soft-excitation region thus it is considered that the hard-excitation region is very small in low Ic and it is very difficult to find the region. We expect to operate a higher power $\mathrm{CW}$ measurement in the hard-excitation region, but our gyrotrons are limited in power in the $\mathrm{CW}$ operation by the documentation and the specification. Therefore the next trial was carried out by high power pulsed operation following a second method.

\subsection{The Result of oscillation by Va change in the Hard-excitation region (2)}

There is another way to operate in the hard-excitation region, especially if it is applied for the high power pulse operation. That operation is conducted by changing the Va during pulse within 10 seconds under the magnetic field was set at a lower value. This method indicates another way to enter the oscillation in the hard-excitation region [2]. The oscillation region and the relationship with $\mathrm{Va}$ and $\mathrm{Ibc}$ are shown in Figure 5. The applied $\mathrm{Va}$ is changed from a higher value to a lower value by the waveform generator that is programed before starting. It is difficult to determine the waveform of Va by suddenly increasing the anode current (Ia), and the oscillation range is very small in the hard-excitation region. One

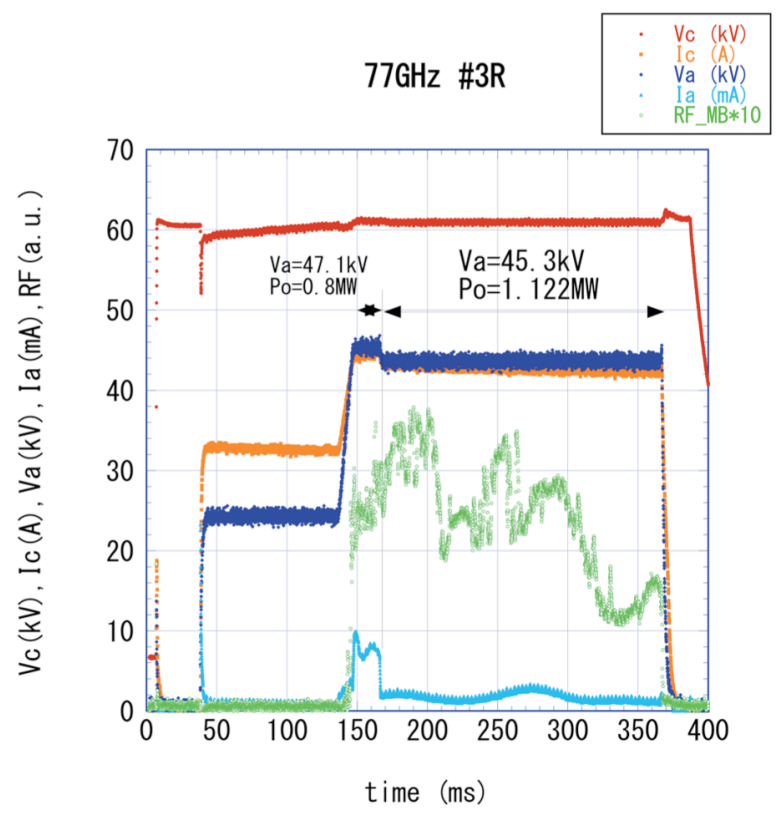

Figure 7. Waveform in the operation of hard-excitation example of this waveform is shown in Figure 6, and applied Va, Ia, and RF signal are shown in Figure 7. Compared to the power which has already been measured at $\mathrm{Va}=46 \mathrm{kV}$, the generated power in $\mathrm{Va}=45.3 \mathrm{kV}$ was recorded approximately $4 \%$ higher (from $1.05 \mathrm{MW}$ to $1.093 \mathrm{MW}$ ). This method was operated repeatedly every $20 \mathrm{sec}$ and could be measured at the same power. In this operational region that can generate over $1 \mathrm{MW}$ at $\mathrm{Ic}=43 \mathrm{~A}$, the oscillation efficiency was improved only $3 \%$.

We attempted to generate more power at the high beam current region at Ic $=60 \mathrm{~A}$ with hard-excitation, but it was impossible due to the suddenly rising anode current that stops operation by the protection limit of the anode over current. And it was very difficult to find a stable oscillation region by this waveform of $\mathrm{Va}$. The region was very small to oscillate, thus we could not find an operational region at hard-excitation.

\section{Two-step Va acceleration}

There is another way to increase the power without entering a hard-excitation region. It is a $\mathrm{Va}$ acceleration done through raising the voltage level twice, or as we call it, two-step Va acceleration [3]. The waveform is seen in beginning of the pulse in Figure 7. It is very effective, valuable, and simple to operate the gyrotrons. Before the operation, the magnetic field is set lower than the normal value and the generation is started by the $\mathrm{Va}$ waveform that includes $\mathrm{Va}=25 \mathrm{kV}$ of no RF in $100 \mathrm{~ms}$. After $100 \mathrm{~ms}$, it can oscillate the higher power compared with the same value of $\mathrm{Va}$. The waveform resembles the state of the hard-excitation region but it might not be different. The phenomenon is caused by charge neutralization in the cavity. The cavity is filled with depressed ions when the body voltage is applied. But the ions are neutralised by

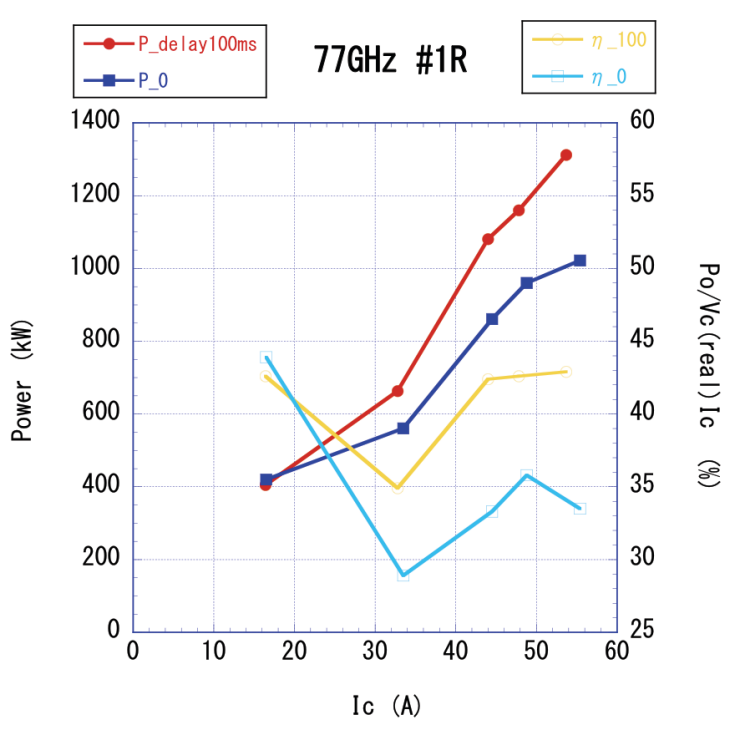

Figure 8. Output power and efficiency depending Ic by a two step operation 
77GHz \#3R

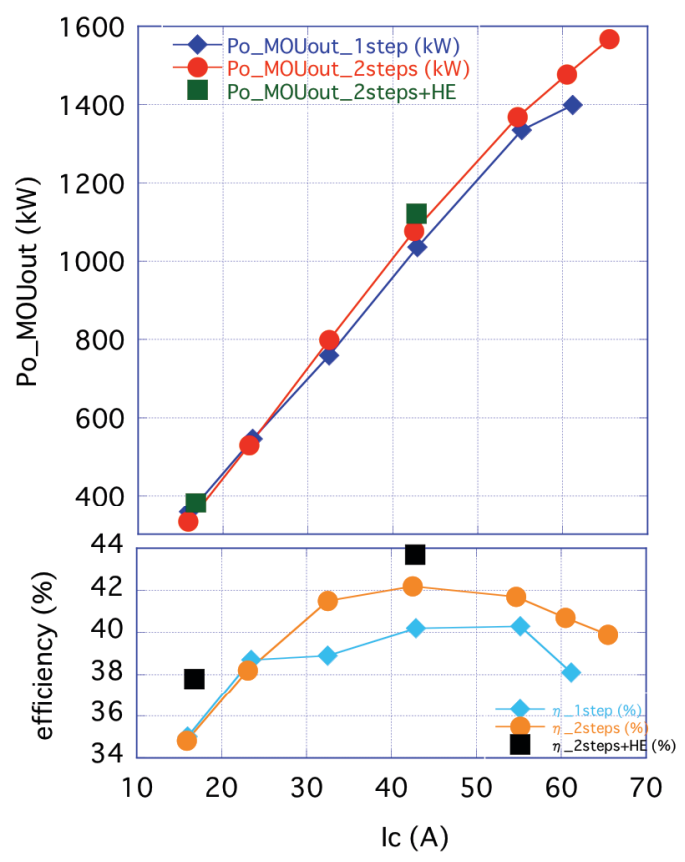

Figure 9. Output power and efficiency depending upon Ic in the region of one-step, two-step, and hard-excitation

first step $\mathrm{Va}$ applied as no RF voltage. Ions are reactivated, and the desired voltage is able to supply. Thus the gyrotron can generate original power. This method used by the anode voltage was originally improved to avoid small operational region by increasing anode current by collector voltage drop at starting pulse. $\mathrm{Vc}$ drop at the beginning of $\mathrm{Va}$ can be seen in Figure 7, too $(\mathrm{t}=40 \mathrm{~ms})$. We could operate higher power region in lower Ibc after this method. The comparison of output power between single and two steps by $77 \mathrm{GHz} \# 1$ is shown in Figure 8. The power difference is clearly observed by Ic between two steps and single step Va. It can also operate steady repetition in long pulse. We combined this technique and hard-excitation to produce more power and efficiency.

\section{The result of oscillation in Hard- excitation and two-step acceleration}

These methods were conducted and achieved the generation of much power and high efficiency. The result of $77 \mathrm{GHz} \# 3$ is shown in Figure 9. The oscillations in the hard-excitation regions are from only two operations in each Ic, but power increase in both of them was confirmed. In particular in the $\mathrm{Ic}=40 \mathrm{~A}$ region, the oscillated power increased approximately $8 \%$ more than conventional operation. The oscillation efficiency also improved from $40.2 \%$ to $43.7 \%$.

\section{Development of ECRH components}

For these experimental operations or usual conditionings, we developed many components for ECRH. We introduce some of them here.

\subsection{The water load with ceramics made of Silicon Nitride (SiN)}

Many kinds of dummy load are used for gyrotron conditioning, and they are distinguished by the materials absorbing RF. For example, bricks, water, stainless steel, titanium dioxide are useful as excellent absorbers. But some disadvantages in these materials exist in the absorption rate of $\mathrm{RF}$, the usage of the vacuum condition, the possibility of CW operation, or the cost. In particular

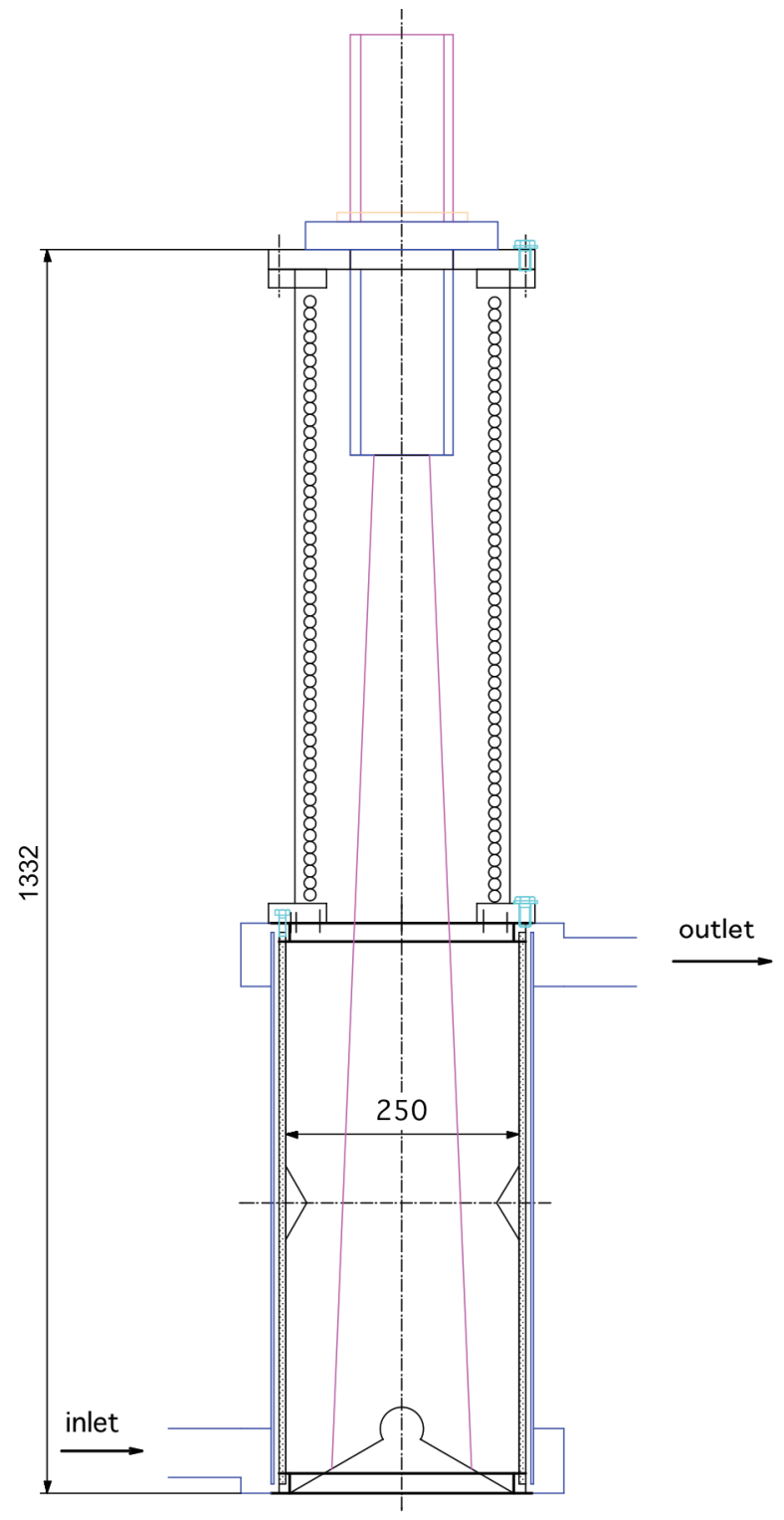

Figure 10. Cross-section of SiN water load 
it is difficult to fix the load once it is broken, or it takes many days to fix, if possible. Therefore, we developed the useful and available load for our purpose of the operation. The cross section of this new load is shown in Figure 10. The load is also needed to measure the accuracy of power and its quick response, thus we selected SiN material, which is also useful for vacuum window, to go through the high power microwave with low loss. Diffracted microwaves in the load pass through the SiN material and are absorbed directly by the cooling water with $1 \mathrm{~m}^{3} / \mathrm{min}$ flowing rate. The load is so sensitive to rising temperature in the cooling water that it is easy to measure the heat even in low output power. The pre-load set on the SiN load absorbs the reflected microwave to prevent it from entering the waveguide.

\subsection{The remote control system in the CW operation and setting the injection patten for the LHD experiments}

Many devices are needed for the operation, but preprogrammed and real-time controllers are very important for the CW operation. The heater voltage device with a pre-programmed and real-time controller is needed to compensate for the decreasing Ic because it occurs soon after the beginning of oscillation and during the $\mathrm{CW}$ by cathode cooling. The heater voltage is set by preprogrammed and controlled until the Ic is saturated. Many kinds of experiments require various injection patterns of ECRH, thus we had to develop an automatically setting pattern of the injection to fulfil the request from the experimental groups. It is very important to quickly input the complicated injection pattern. The direct request of injection timing and duration from the central control room is sent to the control personal computer and is translated to a waveform pattern. The pattern is sent to waveform generator and compiled. It is for the state of being ready to operate. These systems were developed by LabVIEW, and it is easy to recognize the visual injection pattern by Graphical User Interface. We have only three operators, it is necessary to control easily and quickly all the devices because the time interval of the plasma discharge is an only three minutes. These control systems greatly contribute to steady operation.

\section{Summary}

Seven gyrotrons in the ECRH are ready for LHD plasma experiment required from high power pulsed to $\mathrm{CW}$ operation at NIFS. $77 \mathrm{GHz}$ and $154 \mathrm{GHz}$ gyrotrons have been improved in high-power operation to extend their oscillation region through the two-step anode voltage acceleration to contribute to the LHD experiments. Oscillation in the hard-excitation region is also effective for raising the power from the gyrotron without special devices. A gyrotron achieved much power gain and higher efficiency than during normal operation in the soft-excitation region by combining two ways: the twostep $\mathrm{Va}$ control and hard-excitation. Developments in $42 \mathrm{~A}$ of the beam current area are $8 \%$ of power gain and $4 \%$ of efficiency improvement. And also in 15A, $7 \%$ of power gain and $1 \%$ efficiency improvement were achieved. This was conducted by one gyrotron and the effect seems determinate because characteristics of gyrotrons defines their oscillation. Meanwhile, other gyrotrons have the possibility to raise the power and efficiency. We must have the opportunity to try to do. We are developing many components of not only water load and remote control system but also others to contribute to the ECRH system and the LHD experiments. We anticipate that this progress and components will contribute to the LHD experiments and the growth of the ECE and ECRH community.

\section{References}

1. K. Sakamoto, A. Kasugai, K. Takahashi, R. Minami, N. Kobayashi, K. Kajiwara, Nature Phys. 3, 411 (2007)

2. T. Kobayashi, A. Isayama, K. Yokokura, M. Shimono, K. Hasegawa, M. Sawahata, S. Suzuki, M. Terakado, S. Hiranai, F. Sato, K. Wada, J. Hinada, Y. Sato, M. Ohzeki, K. Takahashi, K. Kajiwara, Y. Oda, A. Kasugai, K. Sakamoto, K. Hoshino, S. Moriyama, Nuclear Fusion. 51, (2011)

3. H. Takahashi, T. Shimozuma, S. Ito, S. Kubo, Y. Yoshimura, H. Igami, M. Nishiura, S. Kobayashi, Y. Mizuno, K. Okada, Y. Takita, S. Ogasawara, R. Makino, T. Mutoh, T. Kariya, R. Minami, T. Imai, Plasma Fus. Res. 7, 1205154 (2012) 